Fecha de recepción: febrero 2017 Fecha de aceptación: agosto 2017 Versión final: septiembre 2018

\section{El diseño como modelo de formación emprendedora en educación universitaria}

Carlos Henrique Xerfan do Amaral *, André Ribeiro de Oliveira ${ }^{\star 2}$ e Sandra Mara Nunes Vivote ${ }^{\star 3}$

Resumen: El escenario de la formación emprendedora en Brasil, todavía se presenta como una acción secundaria en las instituciones de enseñanza superior. La complejidad de esta formación pasa por características personales, culturales y técnicas diversas y, desarrollar tales capacidades durante la formación de un profesional es una tarea compleja, reforzada por la fragmentación académica de cada campo específico de actuación.

Con el objetivo de colaborar con un modelo capaz de proporcionar una solución para esta deficiencia de los currículos diversificados y rígidos en el modelo educativo brasileño, este estudio buscó investigar de qué forma el diseño puede contribuir como un recurso objetivo para la formación superior emprendedora. Para ello, el estudio de caso fue realizado en una institución de enseñanza superior brasileña, con característica filantrópica, que se sitúa en la región de la Leopoldina en el Estado de Río de Janeiro.

Palabras clave: emprendedor - enseñanza superior - currículo.

[Resúmenes en inglés y portugués en las páginas 277-278]

${ }^{(*)}$ Fisioterapeuta, Mestre em neurociências (Universidade Federal Fluminense). Doutorando em Design (Escola Superior de Desenho Industrial, Universidade do Estado do Rio de Janeiro). Diretor de Inovação e Novos Negócios (Centro Universitário Augusto Motta).

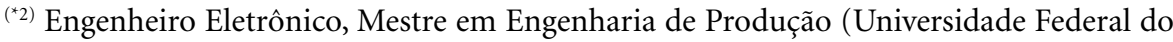
Rio de Janeiro). Doutor em Engenharia de Produção (Universidade Federal do Rio de Janeiro). Professor adjunto da Universidade do Estado do Rio de Janeiro e professor adjunto da Pontifícia Universidade Católica do Rio de Janeiro.

${ }^{* 3)}$ Pedagoga, Mestre em Desenvolvimento Local (Centro Universitário Augusto Motta). Doutora em Sociologia (Instituto Universitário de Pesquisa do Rio de Janeiro). Professora de Empreendedorismo no Centro Universitário Augusto Motta; Psicopedagoga do Núcleo de Apoio Psicopedagógico (UNISUAM). Tutora a distância - Gestão Pública Educacional - Projeto da Secretaria Municipal de Educação do Rio de Janeiro - SME / SENAC. 


\section{Introducción}

El escenario de la formación para emprendedores en Brasil aún fracasa en presentarse de un modo consolidado y, de acuerdo al último reporte del Global Entrepreneurship Monitor, el país carece de programas de entrenamiento que coincidan en proveer la experiencia necesaria para un emprendedor. Podemos observar que la mayoría de los estudiantes universitarios en el país no presentan, durante su entrenamiento, acceso a conocimiento, experiencia y actitudes que los hagan habilidosos para desarrollar nuevos negocios, adquiriendo en su mayoría contenidos dirigidos, específicamente, al mercado laboral. (Dolabela, 1999) La complejidad de la formación para emprendimientos pasa por diversas características personales, culturales y técnicas, y desarrollar estas capacidades durante el entrenamiento de un profesional es una tarea difícil, reforzado por la fragmentación académica de cada campo específico. En 1970, Peter Drucker describió al emprendedor como aquel que "siempre busca cambios, responde a ellos y los explota en una oportunidad".

El modelo mental necesario para el desarrollo de las empresas mismas concierne características cognitivas, como por ejemplo, habilidades creativas superiores, halladas en escuelas multidisciplinares con foco en creación y reflexión de proyectos, presentes en el campo del diseño. Buscando, de este modo, colaborar con un modelo capaz de promocionar ciclos de entrenamiento sobre currículas estrictas y diversas para el modelo educativo brasilero, este estudio propone investigar de qué modo puede contribuir el diseño como un recurso objetivo para la formación empresarial universitaria.

Con este propósito, se dirigió un caso de estudio en una institución universitaria brasilera, de características filantrópicas, localizada en la región Leopoldina en el Estado de Río de Janeiro. La comunidad académica presente en esta institución es representada por un perfil económico y social particular: jóvenes y adultos recientemente ascendidos a la universidad en el país, y que debieran ese ascenso, hasta cierto punto, a políticas públicas y recursos financieros para sostener sus estudios.

Fue utilizado, entonces, la planificación académica del semestre para una asignatura empresarial, usando el diseño como un recurso de enseñanza, con un modelo de negocios basado en el Modelo Canvas, trabajado con estudiantes y profesores de diferentes clases y áreas de entrenamiento, que fueron guiados hacia la creación de negocios reales basados en prototipazo y diseño de objetos a lo largo de un semestre de entrenamiento.

Los datos fueron adquiridos mediante entrevistas llevadas a cabo en muestras grupales luego del cierre del semestre, información recolectada por los profesores basada en el calendario de entregas, registros electrónicos y fotográficos de los proyectos y mediciones de los resultados financieros proyectados por los grupos evaluados en esta acción.

La información ha sido compilada y sugiere que las herramientas del diseño son importantes y deben ser tomadas en cuenta en la formación empresarial, teniendo una percepción positiva de los involucrados y contribuyendo al estímulo al desarrollo creativo, mediante la flexibilidad, la auto-eficacia, la percepción de la habilidad emprendedora y, por consiguiente, en el apoyo a la creación de nuevos negocios, que se presenten como una alternativa a la creación de carrera así como para alcanzar resultados financieros. Futuros estudios colaborarán en un modelo de aplicación más maduro que pueda ser replicado en otras instituciones universitarias. 


\section{Emprendimiento y entrenamiento empresarial}

El mundo ha cambiado radicalmente en el cambio de siglo. Muchas economías se han transformado, se crearon profesiones y un nuevo orden mundial pareció emerger en relación a las ambiciones comerciales y las operaciones globalizadas. Grandes corporaciones han sido creadas por iniciativas individuales o pequeños grupos, a menudo asociadas a compañías que no intentan desarrollar artefactos industriales, sino como simples proveedores de equipos, para que los clientes puedan tener acceso a servicios inimaginados en otros tiempos, especialmente en lo que refiere a cuestiones digitales.

En un cierto modo, nos estamos dirigiendo hacia una nueva revolución industrial, donde la apropiación de los métodos de producción por los emprendedores permite a una persona manejar toda la cadena productiva de un artefacto con varias tecnologías y alcanzar a sus consumidores finales sin tener que lidiar con una industria establecida. Pequeños emprendedores pueden explorar nuevos mercados y desafiar a grandes corporaciones basadas en sus habilidades individuales y otras características empresariales.

El modelo de negocios basado en la web actúa sobre las acciones de inversión de los consumidores para financiar el desarrollo de productos (fenómeno conocido como crow funding), estableciendo una operación mediante la presentación de bajas barreras de presentación en el mercado, rápida innovación e intenso emprendedorismo. (Andrew, 2014) Estos modelos crean posibilidades de trabajo y un diseño de negocios diferente al estatus quo. Empleadores sin un estricto esquema de trabajo y manejo descentralizado, basado en conocimientos y proyectos, con menos burocracia y estructura, sin hierocracia como la estructura soporte de las actividades de coordinación. Un manejo que busca ofrecer ambientes de trabajo informales, con comida saludable disponible a cambio de desempeño, compromiso y rápido reconocimiento. En este movimiento, han emergido nuevos modelos de trabajo y profesiones, y los millenials desafían los sistemas basados en estabilidad y desempeño que fueran el fundamento de los negocios en la época de los baby boomers; un nuevo concepto de "ser exitoso" surge más allá de lo que era entendido como exitoso en las grandes corporaciones globales. En un nuevo escenario, profesionales calificados comienzan a aspirar a la apertura de negocios ya no como algo esencialmente familiar o secundario, sino como su primera opción después de la universidad (o incluso antes que eso). Sin embargo, persisten algunos cuestionamientos al respecto: ¿cómo entrenar profesionales para profesiones que no estamos siquiera seguros que vayan a existir? ¿qué modelo puede realmente ser aplicado en diferentes cursos e instituciones educativas?

Para entender el proceso de construcción de la naturaleza emprendedora y las habilidades requeridas para que un estudiante piense realmente en los negocios como una parte importante de su tarea y la parte instrumental relacionada con las habilidades administrativas y el modelado de productos y servicios componen también las premisas de conocimientos de un emprendedor.

Sin embargo, para despertar la voluntad de emprender, la resiliencia y las actitudes correctas son fundamentales para desarrollar una cultura emprendedora en las personas, usualmente referida con el término poético "brillo en los ojos".

Emprendedores alrededor del mundo parecen reunir una serie de características o ciertas habilidades que parecen constituir la llave para que una persona pueda tener la oportuni- 
dad de aplicar su conocimiento técnico adquirido durante su capacitación en el desarrollo de negocios o proyectos innovadores. Más allá de estas características, destacamos la autonomía, la auto-eficiencia, la propensión a tomar riesgos, la flexibilidad, la creatividad, la atención al mercado y la necesidad de logros, entre otros. (Oosterbeek, 2010)

Para Shumpeter (1985), "el espíritu emprendedor" va más allá de la simple creación de nuevos negocios; está en el afán de implementar algo nuevo. Y este deseo sería responsable de la expansión de la economía y el desarrollo. El autor define dos tipos de individuos: el capitalista, que vive de la economía, administrándola y manejando los pasos de la producción al consumo del activo; y el hombre de negocios, que tendría el espíritu innovador y alimentaría el desarrollo. La concepción del hombre de negocios de Schumpeter es más cercana al concepto de emprendedor moderno:

Entendemos por 'desarrollo', por lo tanto, sólo los cambios en la vida económica que no fueron impuestos, sino que vienen desde dentro, por iniciativa propia. Si se llega a la conclusión de que no existen cambios de este tipo en su propia esfera económica y que el fenómeno que llamamos desarrollo económico se basa, en la práctica, en el hecho de que los datos cambian y la economía se adapta continuamente a él, entonces podríamos decir que no hay desarrollo económico. Nuestro objetivo es decir que el desarrollo económico no es un fenómeno que se pueda explicar económicamente, sino que la economía, sin desarrollo en sí misma, es arrastrada por los cambios del mundo que la rodean, y las causas y, por lo tanto, la explicación del desarrollo, deben ser buscados fuera del grupo de hechos descritos por la teoría económica. (...) El desarrollo, en el sentido que lo tomamos, es un fenómeno distinto, totalmente extraño a lo que se puede observar en el flujo circular o en la tendencia al equilibrio. Es un cambio espontáneo y desigual en los canales de flujo, una perturbación del equilibro que altera y desplaza permanentemente el estado de equilibrio existente anteriormente. (Schumpeter, 1985, p. 47)

Estos estudios relacionan el término emprendimiento con la innovación, la transformación y la audacia. Es vista como una traducción libre que se hace de la palabra emprendimiento que sostiene las ideas de la innovación. El profesor Fernando Dolabela, en su propuesta de "educación en emprendimientos" va más allá.

Para el autor, el término emprendedor implica una forma de ser, una idea del mundo, una forma de relacionarse con los demás. El empresario es un insatisfecho que transforma su infelicidad en descubrimientos y propuestas positivas para sí mismo y para los demás. Él es el actor principal y el autor de sí mismo y principalmente de la comunidad en la que vive. (Dolabela, 2006, p. 26)

En otras palabras, Dolabela busca elementos subjetivos que pueden transformar el significado del término emprendedor en una acción objetiva, acercando al emprendedor al término empoderamiento. (Dolabela, 2003, p. 24)

Dolabela también se conduce hacia una visión más conductista, a un enfoque cognitivo de la político, la visión y el significado. "Es empresario, en cualquier área, alguien que sueña y busca convertir su sueño en realidad". 
Argumenta que cualquier persona puede ser un emprendedor, pero necesita algunas condiciones, como un entorno favorable, democracia, cooperación y una estructura de poder encaminada hacia el diseño en red, con el objetivo de determinar y liderar en este contexto. En una sociedad donde todos pueden ser hombres de negocios, dependiendo sólo de, como señala Richar Sennet (2006), ser entrenado, creativo y talentoso. El autor afirma que esta mentalidad vinculada al sistema económico, cada vez más competitiva, es incapaz de integrar a la mayoría de los trabajadores al mercado de trabajo, ya que, a pesar de invertir en un sistema educativo que pretende actuar en los temas de formación, la creatividad, las características individuales y la capacidad de crear algo nuevo, el mercado de trabajo se está estrechando continuamente y volviendo más competitivo.

El autor sostiene que esta mentalidad ligada al sistema económico, crecientemente competitivo, es incapaz de integrar a la mayoría de los trabajadores al mercado de trabajo, en la medida en que, a pesar de la inversión en un sistema educativo que busca actuar sobre la formación misma, promoviendo la creatividad, las características individuales y la capacidad de crear algo nuevo, el mercado de trabajo se está encogiendo continuamente y volviéndose más competitivo.

La economía de las capacitaciones sigue dejando a la mayoría de las personas de lado: lo que es peor, el sistema educativo genera una gran cantidad de jóvenes graduados imposible de emplear, al menos en el campo para el cual han sido formados (...) la sociedad de habilidades podría necesitar sólo una relativamente pequeña cantidad de capacitados con el mayor talento, especialmente en los sectores avanzados de altas finanzas, tecnología avanzada y de servicios sofisticados. (Sennett, 2006, pp. 82-83)

El autor nos advierte sobre las propias contradicciones entre el discurso pedagógico educativo y la estructura del mercado de trabajo actual. Cuanto más talentos jóvenes se forman como trabajadores formados y expertos, menos son incorporados para desarrollar sus tareas. Más y más ingenieros, managers, economistas, físicos, abogados, entre otros, han estado ocupando puestos de trabajo menos nobles, por ejemplo, aquellos que no requieren el conocimiento adquirido en la etapa de formación universitaria. En palabras de Sennett (2006):

Usualmente, a un joven profesional recientemente salido de la universidad, le lleva de tres a cuatro años comprender para qué es útil lo que estudió. Profundizar la capacidad mediante la práctica, se contrapone a las instituciones que quieren que la gente haga muchas tareas diferentes en un corto plazo. (Sennet, 2006, p. 100)

¿Cuál es la salida al estancamiento que implica integrar una masa de trabajadores jóvenes en un mercado de trabajo, cada vez más competitivo? Tal vez el empoderamiento de las personas, valorando el concepto de emprendedor individual es, de hecho, parte de la solución. Para que cualquiera pueda ser su propio manager. Dueños de su propio negocio. Sin embargo, retomando a Sennett (2006, p. 86), "la esperanza de convertirse en un pequeño hombre o mujer de trabajo, permanecerá para muchos sólo como una esperanza”. 
A pesar de comprender la contradicción entre el discurso y la vida práctica, señalada por el autor, podemos notar un incremento de la inversión en educación para emprendedorismo y el liderazgo de los sujetos y, por lo tanto, su naturalización, lo que llama la atención de los mentores.

El emprendedorismo se parece cada vez más a un proceso en desarrollo que a un evento único, como sostienen Baron y Shane (2011, p. 16). En cada etapa del proceso, todas las variables de cada nivel individual, interpersonal o grupal, así como las variables del nivel social, juegan un rol.

Esta visión demanda la comprensión del proceso, cómo se desarrolla y los diferentes factores que determinan el éxito de los emprendedores y, al mismo tiempo, busca proporcionar a los mismos emprendedores, información práctica útil para desarrollar habilidades que le permitan lograr sus objetivos.

Para Vanilda Paiva (2002, p. 53), ya no es posible resaltar sólo la educación como una vía para el ascenso social, en la medida en que existe una desigualdad acumulativa. Es preciso, de acuerdo a los conceptos de ideología "sistémico-organizacional", más que el conocimiento adquirido mediante el sistema educativo. Se necesitan competencias individuales y voluntad de ganar o, como argumenta Paiva (2002, p. 56), "gestiones y cualidades adquieren más peso que la competencia específica”. En otras palabras, es necesario que el empleado tenga una actitud de emprendedor, de manera de estar preparado no para el trabajo, sino para la vida en su conjunto.

El conocimiento no es suficiente, pero sí el interés, la motivación, la creatividad. No se trata sólo de calificar para el trabajo per se, sino para la vida en la que se inserta el trabajo, con una flexibilidad y un alcance suficiente para afrontar el trabajo, la desocupación y el auto-empleo y moverse, con habilidad, en torno a las diferentes "eras" de las tecnologías, con la posibilidad de comprender y utilizar las máquinas más modernas y afrontar sus múltiples consecuencias en la vida social y personal. (Paiva, 2002, p. 56)

En este sentido, Paiva (2002) convalida la perspectiva señalada por Sennett (2006) y otros autores donde establecen la existencia de una inversión en educación para emprendedores para formar a los sujetos contemporáneos. El emprendedor es usualmente asociado a la innovación y al aprovechamiento de oportunidades. Para Shane (2005), la mayoría de los libros que conciernen a emprendedorismo se vinculan a las cuestiones operacionales relacionadas a cómo abrir un nuevo negocio o la identificación de las características más importantes del emprendedor.

Para Etzkowitz (2003), la Universidad tiene un rol fundamental en la diseminación del emprendedorismo. Para él, la universidad tiene la habilidad de crear una dirección estratégica, elaborando metas académicas claras y convirtiendo el conocimiento generado en la universidad en un potencial económico.

Asimismo, considera la universidad como un ambiente abierto para la innovación (una incubadora natural) para la concentración del capital intelectual, donde los estudiantes son potenciales emprendedores y provee estructuras de soporte para que tanto profesores como estudiantes inicien nuevos negocios: intelectuales, comerciales o asociados. 
Wissema (2008) señala las siguientes características de una Universidad emprendedora:

1. La explotación del know-how se convierte en uno de los principales objetivos, ya que las universidades son vistas como la cuna de la nueva actividad empresarial además de las tareas tradicionales de investigación y educación;

2. Operan en un mercado competitivo a nivel internacional. Compiten activamente por obtener los mejores académicos, estudiantes y contratos de investigación de parte de la industria;

3. Universidades interconectadas, colaborando con la industria, el sector de I+D privado, el sector financiero, proveedores de servicios profesionales y otras universidades mediante un carrusel de conocimientos (ver más abajo);

4. La investigación deberá ser, a la larga, transdiciplinaria o interdisciplinaria;

5. Multiculturalidad, con un rango diverso de estudiantes.

6. Cosmopolitismo, operando en un escenario internacional.

7. Menor dependencia de la regulación estatal y, en el extremo, podría estar completamente desconectado del estado si la financiación directa se sustituye por la financiación indirecta.

Fayolle y Redford (2014) destacan el rol de la universidad en el proceso de capacitación para emprendedorismo. En su opinión, la universidad debe desarrollar e implementar temas y otras actividades que fomenten las actitudes de los estudiantes hacia el emprendimiento. Andrade and Torkomian (2001), también destacan que hay una necesidad de crear un modelo brasilero de educación en emprendedorismo que comprenda los valores culturales, sociales, políticos y económicos del país.

La convergencia de estudios de investigación en campo práctico de desarrollo de innovación y diseño de proyectos, es al mismo tiempo, importante y desafiante. Realizar estudios de naturaleza científica en ámbitos prácticos y dinámicos como el emprendimiento que respondan a un rigor metodológico y, al mismo tiempo, hacer propuestas de generación posibles, constituye un reto metodológico respecto a los enfoques tradicionales aplicados a las ciencias naturales.

\section{La enseñanza del emprendedorismo desde la perspectiva del diseño}

El dinamismo de la prescripción respecto de enfoques específicos para esta población, así como el alineamiento con los criterios científicos adecuados, usualmente no se presenta de manera efectiva, alejando el modelo de investigación del mundo real de las organizaciones y, como resultado, la generación de una propagación generalista, unilateral y aislada del conocimiento, con defectos multidisciplinares que se hacen evidentes cuando se intenta generalizar los conocimientos adquiridos. Es necesario, entonces, (re) pensar las metodologías de investigación que puedan seguir ese movimiento.

La ciencia del diseño fue abordada por el economista norteamericano Alexander Simon en 1996 en su publicación Ciencias de lo artificial, donde las ciencias artificiales se deben preocupar sobre cómo deberían ser las cosas, ya sea para revolver un problema conocido o para diseñar algo que aún no existe. La ciencia natural es un cuerpo de conocimientos so- 
bre objetos o fenómenos del mundo, que conciernen a cómo se comportan e interactúan entre sí, lo que significa que intenta explicar cómo son las cosas. Las ciencias artificiales, por su parte, están interesadas en cómo las cosas deberían ser, para alcanzar metas y trabajar adecuadamente:

La discusión sobre la ciencia del diseño tuvo lugar cuando se identificó una brecha en torno al empleo único y exclusivo de las ciencias tradicionales en la realización de ciertas investigaciones. Las investigaciones destinadas a estudiar el proyecto, la concepción o incluso la resolución de problemas no pueden sostenerse exclusivamente con el paradigma de las ciencias naturales y sociales. (Dresch, 2015)

En las ciencias explicativas, el producto habitual de investigación es el modelo causal, en donde una o más variables dependientes son explicadas por uno o más variables independientes. El conocimiento sobre esas variables puede ser usada para predecir el comportamiento de variables dependientes. En lo que concierne a las ciencias del diseño, el producto usualmente es la prescripción, o como dice Bunge (1967), la "regla tecnológica". Esto puede ser definido como una muestra conveniente del conocimiento general, relacionada con una interferencia o un artefacto con un resultado deseable o que pueda aplicarse en un campo específico. (Van Aken, 2004)

El sustento de las dinámicas de trabajo se basa en la lógica CIMO, que se compone del acrónimo de las palabras "Contexto", "Intervención", "Mecanismo(s)" y "Outcomes" (Resultados), y está construido con la lógica de que si tenemos contextos problemáticos, usaremos un tipo de intervención para reforzar la generación de mecanismos de ideas para así crear nuevas perspectivas. (Denyer, 2008)

Mientras las publicaciones en el ámbito de la enseñanza universitaria están, en su mayoría, focalizados en evaluaciones descriptivas, y no prescriptivas, el razonamiento de acciones prescriptivas con una estructura que permita la construcción de conocimiento en naturaleza teórica (módulo 1), naturaleza práctica (módulo 2) (Gibbons, 1994). La misión principal de una ciencia del diseño es desarrollar conocimiento que pueda ser usado por profesionales en determinadas áreas para diseñar respuestas a sus problemas prácticos. Comprender la naturaleza y las causas de los problemas puede ser de gran ayuda en el diseño de soluciones. Sin embargo, la ciencia del diseño no sólo se limita al entendimiento, sino también al desarrollo del conocimiento sobre las ventajas y desventajas de soluciones alternativas. (Van Aken, 2005)

En la búsqueda de aplicar la lógica CIMO al campo práctico en entornos educativos desafiantes, sugerimos que el diseño podría ser el catalizador de nuevas experimentos y experiencias de emprendedorismo que puedan brindar a los estudiantes las premisas volcadas en el plan de estudios.

Ampliamente discutida en la actualidad, la metodología de Design Thinking ha contribuido en la construcción de proyectos innovadores focalizados en escenarios prácticos y que cuentan con la máxima aceptación de los targets, dirigiéndose a un público a menudo compuesto de personas sin una formación específica en el diseño, que es llevado a un modelo mental de pensamiento de diseñador. 
Este marco teórico ha sido ampliamente usado alrededor del mundo. Esencialmente, Tim Brown, de la compañía de diseño IDEO, introdujo otro paradigma a la realidad de los proyectos de diseño, empujando a la comunidad en un movimiento para pensar más allá de los artefactos y los proyectos y abordar tres premisas: que los diseñadores deben involucrarse más en los aspectos sociales vinculados con sus proyectos, que el diseño es un esfuerzo colaborativo y que el proceso debe incorporar stakeholders y, finalmente, que las ideas deben tornarse tangibles mediante prototipos. (Bjögvinsson, 2012)

El rol del diseñador ha sido tradicionalmente vinculado con la creación y funcionalidad de formatos como imágenes, información, objetos y productos y, el diseño en su tradición formal, como el producto final de un proceso de innovación o desarrollo, usualmente orientado a "poner una idea en un bonito empaque" (Brown, 2008). Obviamente, no habría tiempo de consolidar toda la capacitación necesaria para formar a un diseñador que se superponga los temas de un estudiante de Derecho, por ejemplo.

El Design Thinking debería funcionar como un puente entre el modelo CIMO y la redacción de propuestas de diseño, siguiendo las etapas organizadas por Tim Brown en la empresa de diseño IDO: Empatizar (aprendiendo sobre los clientes), Definir (creando un punto de vista basado en sus necesidades), Idear (a través de diversas soluciones creativas), Crear prototipos (construyendo representaciones de las ideas) y Testear (compartiendo el prototipo para obtener feedback e introducir mejoras). Este sería entonces el soporte para colaborar en la construcción de la mentalidad de un diseñador, enseñando a los estudiantes a pensar en un modo proyectual, como lo haría un diseñador, canalizando los prototipos en un modelo "hágalo usted mismo" y valorando la cultura creadora de los tiempos presentes.

Sin embargo, debe considerarse una cuestión importante: ¿cómo desarrollar esta actividad en un contexto complejo en el que las clases están compuestas por estudiantes y profesionales que no fueran diseñadores? La totalidad de los grupos de estudiantes ha tenido una cantidad de aproximadamente 2700 individuos, distribuidos en clases de diferentes áreas formativas (en un mismo grupo de seis estudiantes pudimos encontrar de áreas completamente distintas y cursos de pregrado aleatorios) totalizando 450 modelos de negocio a ser desarrollados. Para hacer posible el desarrollo de estas actividades en un semestre, con sólo un encuentro semanal con el profesor y con un mínimo de recursos permitidos fue, en efecto, desafiante. La siguiente sección explica cómo el trabajo de campo fue desarrollado.

\section{Trabajo de campo}

Analizar y reflexionar críticamente sobre los programas y proyectos de educación en emprendedorismo que se propone en sus metas educativas y pedagógicas, para despertar las características del emprendedorismo y sus implicancias con las dinámicas de la sociedad capitalista, fueron los puntos de discusión de esta investigación. Asimismo, presentamos información empírica proveniente del campo de investigación, realizada con una universidad privada de la ciudad de Río de Janeiro, Brasil, que contribuyó con una serie de datos cualitativos y estudios concretos sobre los desafíos de la enseñanza del emprendedorismo en el ámbito universitario. 
La investigación partió de una premisa inicial que sostiene que las características del emprendedorismo pueden contribuir al desarrollo de los estudiantes, más allá de sus limitaciones provenientes de escenarios complejos que se expresan en su vida cotidiana en una desmotivación por sobrellevar cualquier proyecto. Se sugiere, entonces, un modelo de formación emprendedora que pueda construir nuevos puntos de vista de los estudiantes basada en un trivium entrepreneur que reúna los factores de autosuficiencia, práctica creativa y pensamiento empresarial.

En este sentido, la conexión entre los negocios y la actividad de diseño adquiere relevancia. Ambos términos refieren a diferentes acciones y campos de conocimiento, pero comparten la naturaleza prescriptiva de ofrecer soluciones a los problemas o demandas sociales. Con el objetivo de ofrecer un modelo de negocios, las tareas de construcción de artefactos deberían, entonces, fijarse como meta la creación de un MVP (producto mínimo viable). El MVP es una versión de un producto que aún carece de muchas características que resultan ser esenciales en etapas más maduras de desarrollo, pero la creación de MVP, además de exigir un mínimo esfuerzo, permite medir su impacto como propuesta. Sin embargo, es importante considerar que la evaluación de un MVP no debe ser realizada únicamente por personas que conocen el producto, sino por clientes potenciales. (RIES, 2011)

El último eje principal de este trabajo reúne a los MVP y prototipos creados desde la propuesta de negocio a través del modelo CANVAS propuesto por Alexander Osterwalder. Este modelo es una visión más pragmática y práctica de la elaboración de planes de negocio en forma reducida, que permite una significativa reducción de tiempo y una construcción colaborativa entre los miembros de un grupo de trabajo, mediante la implementación de acciones de ejecución de negocios para validar los modelos planificados y haciendo posibles ciclos de mejora. El desarrollo de este modelo se produce en bloques que siguen una secuencia lógica sugerida por el autor: proposiciones de valor, segmentos de clientes, canales, relaciones con los clientes, flujos de ingresos, recursos claves, socios claves, actividades claves y estructura de costos.

Basados en estas premisas, entendemos como alternativa el desarrollo de negocios que puedan dar lugar a prototipos comercializables, manteniendo el foco en proposiciones de diseño y considerando la distinción esencial entre el conocimiento para resolver problemas teóricos y el conocimiento para resolver problemas prácticos.

El estudio fue realizado en un centro universitario localizado en la región conocida como Leopoldina en el estado de Río de Janeiro, Brasil, escenario para la prescripción de soluciones. En la institución referida, algunos factores colaboraron en esta acción, entre ellos la existencia de una currícula sobre emprendedorismo y un programa obligatorio en todos los cursos de pregrado y grado, con un enfoque multidisciplinario y práctico, además de contar con un núcleo de apoyo al emprendimiento y un espacio de trabajo preparado para ayudar a los profesores y estudiantes. Las características socioeconómicas de los estudiantes comprender mayoritariamente las clases C y D que esencialmente manifiestan en sus discursos el deseo de obtener una formación para lograr ascensos laborales o participar en licitaciones. Para articular todas estas prácticas, los estudiantes fueron distribuidos en clases que recibieron un conjunto de materiales teóricos en una plataforma online, así como actividades de tutoría en el aula con los profesores de las asignaturas. 
Grupos de seis estudiantes emprendedores fueron capacitados para estructurar un negocio, combinando estudiantes de diferentes cursos de las áreas de ingeniería, salud, humanidades, ciencias biológicas y sociales, principalmente en fases iniciales de sus respectivos campos y focalizados en obtener un diploma, sin conocimientos, a priori, del valor del emprendedorismo en sus vidas profesionales, académicas y personales.

Como un modo de manejar la lógica CIMO, se decidió emplear la metodología del Design Thinking como vía para la comprensión de las necesidades contextuales y el incremento de las oportunidades, validando así las ideas innovadoras.

Los calendarios de actividades planificadas para el semestre, tuvieron tres eventos de referencia que establecieran etapas de evaluación (que eran actividades de prototipado y validación) y un evento de cierre en el que todos los grupos debían participar en una feria empresarial dentro de la institución. Los emprendedores también debían confiar en sus canales de comunicación y sus habilidades de ventas para incrementar las posibilidades de adquisición de sus artefactos.

Buscando guiar la producción de prototipos, se estableció una temática generadora para que los estudiantes pudieran tener un norte y focalizaran sus energías en un modo más productivo, dejando de lado el mundo abstracto en pos de la materialización. La temática relacionada fue el reciclaje de chatarra electrónica.

Para la evaluación de la iniciativa se realizó una reflexión en la que se registraron todos los pasos el desarrollo de negocios CANVAS (Osterwalder y Pigneur, 2010). Fueron seleccionados aleatoriamente entre los docentes mentores de las empresas, 40 proyectos de evaluación, en diferentes clases y campi. Los proyectos seleccionados como muestra tendrían su participación en el evento de cierre.

\section{Resultados}

Los 38 proyectos evaluados hicieron fluir una suma de mil novecientos cincuenta y nueva reales en operaciones comerciales durante el festival de validaciones, con márgenes y volúmenes de ventas variados, y todos los beneficios de las ventas se dividieron en acciones entre los socios. A pesar de que todos los proyectos del grupo de muestra terminaron los modelos de acuerdo con el enfoque Canvas, alguno proyectos estaban mejor estructurados y eran más comercializables que otros, con un margen de beneficios más satisfactorio. De los 476 proyectos desarrollados durante el semestre, 384 desarrollaron todas las etapas en esquemas apropiados y todos los proyectos fueron presentados de algún modo. Sin embargo, tres grupos desarrollaron sus negocios sin el modelo Canvas. En los grupos de muestra seleccionados, todos concluyeron mínimamente sus actividades de llenado del lienzo y modelado de sus negocios. En este grupo de muestra, aproximadamente el 10\% falló en la obtención de beneficios con las ventas de sus proyectos durante la feria de validaciones, ya sea por ausencia de clientes o por un desarrollo equivocado de la estructura del negocio, señalando los defectos en el desarrollo de negocio y un 5\% tuvo pérdidas con la operación, experimentando dificultades en la transposición del plano teórico a la realidad. Otra información relevante relacionada con los proyectos está conectada con el modelo Canvas. Ponderando el promedio entre los resultados alcanzados, se logró el valor de 
$\mathrm{R} \$ 50,25$ reales entre las empresas. Sobre la base de esta cantidad, se identificaron en el grupo evaluado once empresas que alcanzaron valores superiores al promedio, que se suman a aproximadamente el $30 \%$ de los grupos estudiados.

Un dato relevante de este proyecto fue el impacto del modelo de producto y la apropiación del diseño como un recurso importante durante el bosquejo de los prototipos. En los grupos evaluados, la relación ente los costos de producción, el margen de ganancia y la reventa parecía ser determinante para obtener resultados positivos. (Ver Tabla 1)

El diseño canalizado a través del enfoque "hágalo usted mismo" trajo a la luz datos importantes. Algunos grupos informaron tener problemas relacionados con la creación de prototipos comercializables, ya sea por falta de aptitud y habilidad, dificultad para desarrollar actividades creativas o la necesidad de infraestructura o un espacio para prototipado en la institución. Sin embargo, la excelencia de la ejecución o la innovación en el producto resultaron ser los dos factores críticos para el éxito de los negocios creados.

Una parte de las dificultades encontradas por los estudiantes estuvo asociada al desarrollo de prototipos basados en el modelo propuesto y algunos informes argumentaron que los temas abiertos podrían promover resultados más factibles. Los profesores de las asignaturas informaron que el modelo era apropiado para el programa propuesto y advirtieron que las mejoras podrían relacionarse con la apertura de temáticas de generación de temas más amplios y que la parte más compleja era, de hecho, involucrar a los estudiantes en el desarrollo de la actitud de un emprendedor.

\section{Conclusiones y recomendaciones para futuros trabajos}

Aún es posible teorizar acerca de la importancia del modelado y el diseño de prototipos, una vez que el rendimiento de algunos productos fue superior al de otros. Evaluar el impacto del diseño y el uso del Design Thinking en el diseño de proyectos, es un objetivo futuro que puede aportar datos relevantes para otras propuestas.

Éstas y otras iniciativas desafiaron a los estudiantes que, en sus informes, se sentían más motivados, capaces de llevar adelante algo innovador y dispuestos a cumplir sus sueños. Espontáneamente, algunos informes de los estudiantes en las redes sociales aportaron información significativa relacionada con los objetivos propuestos por la iniciativa: "Nunca me sentí capaz de realizar mi propio negocio. ¡Terminé la materia con nuevas órdenes e intentaré seguir adelante con la iniciativa!". No obstante, debe destacarse que no todos los estudiantes lograron cumplimentar todas las etapas propuestas en el modelo.

En el modelo sugerido, la existencia de un espacio equipado para el desarrollo de actividades de creación en los modelos de un taller o un fablab, parece ser importante para la ejecución de actividades. Muchos estudiantes afirmaron que la dificultad de contar con los recursos apropiados tornaba más difícil la concepción de prototipos. Otro punto importante se relacionó con el uso del lienzo Canvas como marco, ya que el tiempo para elaborar los proyectos es considerado corto por los estudiantes. Mientras tanto, el diseño como herramienta de formación emprendedora demostró tener una gran capacidad como catalizador del potencial creativo del ejecutante, estimulando a los estudiantes a convertirse en ejecutantes y generar (re) interpretaciones del mundo en el que viven. 


\begin{tabular}{|c|c|c|c|}
\hline Nombre del negocio & $\begin{array}{l}\text { Margen de } \\
\text { ganancia esperado }\end{array}$ & Ganancia & Canvas-volumen \\
\hline Arte Biju & $50 \%$ & $\mathrm{R} \$ 17,00$ & 23. \\
\hline Reciclagem Consciente & $140 \%$ & $\mathrm{R} \$ 37,50$ & 28 \\
\hline Beautiful House & $50 \%$ & $\mathrm{R} \$ 196,00$ & 9 \\
\hline E-ACESSîRIOS & $78 \%$ & $\mathrm{R} \$ 0,00$ & 17 \\
\hline \#Tecnoe-Pet & $41 \%$ & $\mathrm{R} \$ 41,00$ & 23 \\
\hline TECNOEDUCANDO & $100 \%$ & $\mathrm{R} \$ 15,00$ & 18 \\
\hline Sucata Inovadora & $52 \%$ & $\mathrm{R} \$ 11,20$ & 9 \\
\hline Porta-retrato de Teclado & $62 \%$ & $\mathrm{R} \$ 7,55$ & 0 \\
\hline ArtDecor Empreendedorismo & $100 \%$ & $\mathrm{R} \$ 137,00$ & 12 \\
\hline VIRTU@L DECORAÇÕES & $20 \%$ & $\mathrm{R} \$ 53,54$ & 9 \\
\hline Cyber Trufas & $45 \%$ & $\mathrm{R} \$ 250,00$ & 10 \\
\hline Felinas Luminłrias & $\mathrm{R} \$ 0,5$ & $\mathrm{R} \$ 39,00$ & 16 \\
\hline Reciclando Momentos & $60 \%$ & $\mathrm{R} \$ 5,27$ & 9 \\
\hline N@turale Books & $125 \%$ & $\mathrm{R} \$ 15,48$ & 9 \\
\hline TrashToy & $140 \%$ & $R \$ 43,00$ & 12 \\
\hline Re Utilitec & $\mathrm{R} \$ 0,56$ & $\mathrm{R} \$ 56,00$ & 9 \\
\hline Recycling & $\mathrm{R} \$ 0,54$ & $\mathrm{R} \$ 65,43$ & 17 \\
\hline EcoTech & $75 \%$ & $\mathrm{R} \$ 40,00$ & 23 \\
\hline Eletro Moda & $79.58 \% / 27.9 \%$ & $\mathrm{R} \$ 25,80$ & 9 \\
\hline X TIME & $\mathrm{R} \$ 25,9$ & $\mathrm{R} \$ 15,40$ & 11 \\
\hline CDecorados & 1 & $\mathrm{R} \$ 48,00$ & 58 \\
\hline arte sustentavel & $65 \%$ & $\mathrm{R} \$ 2,50$ & 0 \\
\hline Ecotec & $29.0 \% / 13.5 \% / 80.1 \%$ & $\mathrm{R} \$ 57,50$ & 18 \\
\hline Avant & $\mathrm{R} \$ 0,37$ & $\mathrm{R} \$ 10,00$ & 0 \\
\hline Luminous K7 & $\mathrm{R} \$ 0,3$ & $\mathrm{R} \$ 30,00$ & 9 \\
\hline E luxo & $235 \%$ & $\mathrm{R} \$ 3,00$ & 17 \\
\hline ReciclART & $58 \%$ & $\mathrm{R} \$ 24,00$ & 11 \\
\hline Recycle Tech Design - LUMINUS-R & $\mathrm{R} \$ 21,42$ & $\mathrm{R} \$ 30,00$ & 1 \\
\hline Ecobijoux & 3 & $\mathrm{R} \$ 11,20$ & 12 \\
\hline 'Ascenda sua consciência' & $50 \%$ & $\mathrm{R} \$ 9,00$ & 21 \\
\hline Recicla Digital & $100 \%$ & $\mathrm{R} \$ 16,00$ & 8 \\
\hline BIO MAIS & $\mathrm{R} \$ 20,83$ & $\mathrm{R} \$ 102,00$ & 17 \\
\hline lixo eltronico & $40 \%$ & $\mathrm{R} \$ 0,00$ & 1 \\
\hline box & $100 \%$ & $\mathrm{R} \$ 70,00$ & 18 \\
\hline house clock & $75 \%$ & $\mathrm{R} \$ 15,00$ & 8 \\
\hline Drink's House & $38 \%$ & $\mathrm{R} \$ 18,00$ & 8 \\
\hline FloppyDisk & $200 \%$ & $\mathrm{R} \$ 70,00$ & 23 \\
\hline Animal Care & $200 \%$ & $\mathrm{R} \$ 330,00$ & 8 \\
\hline
\end{tabular}

Tabla 1. Información sobre las empresas generada durante la presentación grupal en el semestre. 
Cabe destacar que la presencia más efectiva de los diseñadores en el despliegue de la materia podría impulsar estos resultados. Dentro del ámbito institucional, el tema del emprendimiento en cuestión es obligatorio para todos los cursos y forma parte del segundo semestre de los programas de pregrado. Es necesario obtener más información para poder establecer cuál es la mejor opción: si desarrollarlo en el inicio de los cursos para la construcción temprana de una formación mental de empresario o desarrollarlo en semestres más avanzados donde la madurez de los temas de los cursos es mayor.

En el escenario en el que la institución se encuentra inserta, las prácticas adoptadas que apuntan a la formación emprendedora entendemos que siguieron un modelo válido para cumplir su rol en la atención y el compromiso de los estudiantes participantes en una sociedad capitalista, y que el aula de la universidad analizada no está al margen de esta realidad.

Retomando la hipótesis, observamos que el fortalecimiento de las características emprendedoras puede contribuir al desarrollo de los estudiantes. Una segunda hipótesis se centró en la propuesta de un modelo de formación de un trívium entrepreneur que refuerza en sus prácticas como una alternativa de sostenibilidad, abriendo otras discusiones acerca de hasta qué punto la propuesta incluye a la sociedad o a la cultura del consumidor y, aún así, los prepara para trabajar en una lógica neoliberal. La universidad que sirvió de ejemplo, no está al margen de esta propuesta, sino que desempeña su rol en una sociedad capitalista y busca motivar acciones de desarrollo local en su comunidad.

\section{Bibliografía}

Andrade, R. F. D. y Torkomian, A. L. V. (2001). Fatores de influência na estruturação de programas de educação empreendedora em instituições de ensino superior. Encontro de estudos sobre empreendedorismo e gestão de pequenas empresas, 2, 299-311

Baron, R. A. y Shane, S. A. (2010). Empreendedorismo-uma visão do processo. Cengage Learning Edições Ltda.

Bellotti, F.; Berta, R.; De Gloria, A.; Lavagnino, E.; Dagnino, F.; Ott, M. y Mayer, I. S. (2012). Designing a course for stimulating entrepreneurship in higher education through serious games. Procedia Computer Science, 15, 174-186.

Bjögvinsson, E.; Ehn, P. e Hillgren, P. A. (2012). Design things and design thinking: Contemporary participatory design challenges. Design Issues, 28(3), 101-116.

Brown, T. (2008). Design thinking. Harvard business review, 86(6), 84. Disponible en: http:// web.me.com/deatkins/CIC/Seminar_Schedule _ files/HBR-Timbrown.pdf x

Cardoso, F. H. (1972). Empresário industrial e desenvolvimento econômico no Brasil (Vol. 13). Difusão Européia do livro.

Denyer, D.; Tranfield, D. y Van Aken, J. E. (2008). Developing design propositions through research synthesis. Organization studies, 29(3), 393-413.

Dolabela, F. (2012). O segredo de Luísa: uma idéia, uma paixão e um plano de negócios: como nasce o empreendedor e se cria uma empresa. Sextante.

Dolabela, F. (2003). Pedagogia empreendedora: o ensino de empreendedorismo na educação básica, voltado para o desenvolvimento social sustentável. São Paulo: Cultura. 
Dresch, A.; Lacerda, D. P. y Júnior, J. A. V. A. (2015). Design science research: método de pesquisa para avanço da ciência e tecnologia. Bookman Editora.

Jackson, A. (2014). Makers: The New Industrial Revolution. Journal of Design History, ept048.

Orford, J.; Wood, E.; Fisher, C.; Herrington, M. y Segal, N. (2003). Global entrepreneurship monitor. South African executive report.

Oosterbeek, H.; Van Praag, M. y Ijsselstein, A. (2010). The impact of entrepreneurship education on entrepreneurship skills and motivation. European economic review, 54(3), 442-454.

Osterwalder, A. y Pigneur, Y. (2010). Business model canvas. Self published. Last.

Paiva, V. (2001). Qualificação, crise do trabalho assalariado e exclusão social. A cidadania negada: políticas de exclusão na educação e no trabalho, 1, 49-64.

Ries, E. (2011). The lean startup: How today's entrepreneurs use continuous innovation to create radically successful businesses. Crown Books.

Schumpeter, J. A. (1961). Teoria do desenvolvimento econômico. Fundo de Cultura.

Shane, S. A. (2009). Sobre Solo Fértil: Como identificar grandes oportunidades para empreendimentos em alta tecnologia. Bookman Editora.

Sennet, R. A. (2006). Cultura do novo capitalismo. Rio de Janeiro: Record.

Van Aken, J. E. (2005). Management research as a design science: articulating the research products of mode 2 knowledge production in management. British journal of management, 16(1), 19-36.

\begin{abstract}
The entrepreneurship training in Brazil is still a secondary action in higher education institutions. The complexity of this training goes through diverse personal, cultural and technical characteristics. The developing of such skills during the training of a professional is a complex task, reinforced by the academic fragmentation of each specific field of activity.

Seeking to collaborate with a solution to this deficiency of the diversified and rigid curricula in the Brazilian educational model, this study sought to investigate in what way the design can contribute as an objective resource for the superior entrepreneurial formation. Therefore, the case study was carried out in a Brazilian higher education institution, with philanthropic characteristics, located in the region of Leopoldina, state of Rio de Janeiro.
\end{abstract}

Key words: entrepreneurship - higher education - curriculum.

Resumo: O cenário da formação empreendedora no Brasil ainda se apresenta como uma ação secundária nas instituições de ensino superior. A complexidade desta formação passa por características pessoais, culturais e técnicas diversas e, desenvolver tais capacidades durante a formação de um profissional é uma tarefa complexa, reforçada pela fragmentação acadêmica de cada campo específico de atuação.

Buscando colaborar com um modelo capaz de proporcionar uma solução para esta deficiência dos currículos diversificados e rígidos no modelo educacional brasileiro, este estudo 
buscou investigar de que forma o design pode contribuir como um recurso objetivo para a formação superior empreendedora. Para tanto, o estudo de caso foi realizado em uma instituição de ensino superior brasileira, com característica filantrópica, que se situa na região da Leopoldina no Estado do Rio de Janeiro.

Palavras chave: emprendedorismo - ensino superior - currículo. 